\title{
Chest X-Ray Not Routinely Indicated Prior to the YEARS Algorithm in the Diagnostic Management of Suspected Pulmonary Embolism
}

\author{
Liselotte M. van der Pol ${ }^{1,2}$ Cecile Tromeur ${ }^{1,3}$ Laura M. Faber ${ }^{4}$ Tom van der Hulle ${ }^{1}$ Lucia J. M. Kroft ${ }^{5}$
} Albert T. A. Mairuhu ${ }^{2}$ Albert de Roos ${ }^{5}$ Menno V. Huisman ${ }^{1} \quad$ Frederikus A. Klok ${ }^{1}$

${ }^{1}$ Department of Medicine - Thrombosis and Hemostasis, Leiden University Medical Center, Leiden, The Netherlands

2 Department of Internal Medicine, Haga Teaching Hospital, The Hague, The Netherlands

${ }^{3}$ Groupe d'Etude de la Thrombose de Bretagne Occidentale, Department of Internal Medicine and Chest Diseases, University of Brest, CHRU Brest, Brest, France

${ }^{4}$ Department of Internal Medicine, Red Cross Hospital, Beverwijk, The Netherlands

${ }^{5}$ Department of Radiology, Leiden University Medical Center, Leiden, The Netherlands

TH Open 2019;3:e22-e27.
Address for correspondence L. M. van der Pol, Department of Thrombosis and Hemostasis, Leiden University Medical Center (LUMC), Albinusdreef 2, 2300 RC Leiden, The Netherlands (e-mail: Lm.vanderPol@lumc.nl).

\begin{abstract}
Keywords

- pulmonary embolism

- diagnosis

- chest X-ray

- clinical decision rule

- D-dimer
\end{abstract}

Background The YEARS algorithm was designed to simplify the diagnostic process of suspected pulmonary embolism (PE) and to reduce the number of required computed tomography pulmonary angiography (CTPA) scans. Chest X-ray (CXR) is often used as initial imaging test in patients suspected for PE.

Aim To determine if CXR results differ between patients with confirmed PE and with $P E$ ruled out, and to investigate whether $C X R$ provides incremental diagnostic value to the YEARS criteria that is used for selecting patients with CTPA indication.

Methods This post-hoc analysis concerned 1,473 consecutive patients with suspected PE who were managed according to YEARS and were subjected to CXR as part of routine care. The prevalence and likelihood ratios of seven main CXR findings for a final diagnosis of PE were calculated.

Results A total of 214 patients were diagnosed with PE at baseline (15\%). Abnormal CXR occurred more often in patients with confirmed PE $(36 \%, 77 / 214)$ than in patients without PE (26\%; 327/1,259), for an odds ratio of 1.60 (95\% confidence interval: 1.18 2.18). Only the unexpected finding of a (rib)fracture or pneumothorax, present in as few as six patients $(0.4 \%)$, significantly lowered the post-test probability of PE to an extent that CTPA could have been avoided.

Conclusion The incremental diagnostic value of CXR to the YEARS algorithm to rule out PE was limited. CXR was more frequently abnormal in patients with PE than in those in whom PE was ruled out. These data do not support to perform CXR routinely in all patients with suspected PE, prior to CTPA imaging. received

June 22, 2018

accepted after revision

October 26, 2018
DOI https://doi.org/

10.1055/s-0038-1676812.

ISSN 2512-9465. (c) 2019 Georg Thieme Verlag KG

Stuttgart · New York
License terms

(c) (i) 


\section{Introduction}

The diagnostic management of suspected acute pulmonary embolism (PE) is challenging due to its nonspecific signs and symptoms. The clinical suspicion of PE must therefore be followed by objective testing. Current guidelines recommend applying clinical decision rules to categorize patients in accordance with their pretest probability of PE. ${ }^{1,2}$ In case of non-high probability of PE, D-dimer testing is warranted because PE can be safely ruled out if the D-dimer test result is normal. In case of abnormal D-dimer or high clinical probability, computed tomography pulmonary angiography (CTPA) should be performed. ${ }^{1-3}$ In recent years, attempts have been made to increase the number of patients in whom imaging is not required to rule out $\mathrm{PE}$, for instance by introduction of an age-dependent D-dimer threshold. A recently published strategy is the simple and straightforward YEARS algorithm that includes simultaneous D-dimer and clinical pretest probability assessment and the application of a pretest probability D-dimer threshold. ${ }^{4,5}$ The YEARS algorithm was shown to safely rule out acute PE (failure rate of the overall algorithm: $0.61 \%, 95 \%$ confidence interval [CI]: $0.36-0.96$ ) and reduce the need for CTPA examinations by $14 \%$ compared with the conventional diagnostic strategy. ${ }^{5}$

Chest X-ray (CXR) is a commonly performed test in the initial evaluation of suspected cardiopulmonary disease and has the advantage of being associated with lower radiation exposure than CTPA. ${ }^{6}$ Since 40 to $88 \%$ of patients with PE have mostly nonspecific abnormal CXR findings, CXR cannot be used to confirm and/or exclude the diagnosis of $\mathrm{PE}^{6-8}$ although it may indicate other cardiopulmonary conditions. ${ }^{6}$ Most prevalent abnormal CXR findings in PE patients are cardiomegaly, atelectasis, elevated hemi diaphragm, pleural effusion, pulmonary infarction, and parenchymal areas of increased opacity. ${ }^{6,7,9-12}$ Interestingly, the NICE guideline recommends to start the diagnostic management of patients with suspected PE with a CXR to exclude other conditions than acute PE. ${ }^{13}$ Strong evidence supporting this recommendation is lacking. The aim of the current analysis was to investigate whether a CXR provides incremental diagnostic value to the YEARS algorithm in the diagnostic workup of suspected acute PE.

\section{Methods}

\section{Study Population}

For this post hoc analysis, 1,711 consecutive patients with suspected PE from the YEARS study from three Dutch hospitals, in which a CXR was performed as part of routine clinical care, were evaluated. All patients were managed according to the YEARS algorithm (-Fig. 1). ${ }^{5}$ Exclusion criteria for the YEARS study were treatment with anticoagulants in therapeutic doses initiated $\geq 24$ hours before inclusion, life expectancy less than 3 months, pregnancy, or allergy to intravenous contrast agents. ${ }^{5}$ Patients with confirmed PE were treated with anticoagulants according to international guidelines. Follow-up consisted of a scheduled outpatient clinic visit or telephone interview after 3 months.

\section{Chest X-Rays}

All patients included in this analysis underwent a CXR in the diagnostic workup for suspected PE before they were referred for CTPA. The results of the CXR were reported by the local attending radiologist, who was either a certified radiologist or a resident under supervision of a certified radiologist. For this analysis, CXRs were classified as normal or abnormal. The following abnormalities were recorded: pleural effusion, consolidation, malignancy/mass, congestive heart failure, pneumothorax, (rib) fracture, and atelectasis.

\section{Aims and Endpoints of this Analysis}

The aim of this analysis was to determine the prevalence of CXR abnormalities among patients with suspected PE and to evaluate if CXR results differ in patients with confirmed PE versus patients with PE ruled out. Further aims were to investigate the potential incremental value of performing a CXR routinely in all patients with suspected PE, that is, whether the posttest probability of PE after certain CXR findings would allow for changing the decision to perform CTPA as indicated by YEARS.

The endpoints of this analysis included the odds ratios (OR; with 95\% CI) between the rate of abnormalities on CXR for patients with confirmed PE versus patients with PE ruled out, and for patients with an indication for CTPA according to the YEARS algorithm versus patients without CTPA indication. Furthermore, we assessed the positive and negative likelihood ratios (LRs) for the specific predefined CXR abnormalities mentioned earlier, to calculate the posttest probability for each abnormality.

\section{Statistical Analysis}

To compare the rate of abnormalities on CXR for patients with $\mathrm{PE}$ versus those without $\mathrm{PE}$, an OR with corresponding $95 \% \mathrm{CI}$ was calculated. To evaluate whether the posttest probability of PE changed after the CXR result, positive and negative LR with 95\% CIs were calculated for each different abnormality on CXR and for a normal CXR. The pretest probability was dependent on the PE prevalence, which was calculated in all patients and in patients who were referred for CTPA according to the YEARS algorithm. All analyses were performed using SPSS, version 23.0, Chicago, Illinois, United States.

\section{Results}

\section{Patient Characteristics}

From the 1,711 eligible patients, CXR was not performed in 238 patients for unknown reasons. After excluding these patients, 1,473 were left for analysis. Their mean age was 54 years, $62 \%$ were female, $14 \%$ of patients had COPD, $2 \%$ had chronic heart failure, and $9 \%$ had an active malignancy. Dyspnea was present in $71 \%$ of these patients, $40 \%$ presented with coughing and $74 \%$ with thoracic pain (-Table 1 ). The patients who were managed without CXR had numerical but not significant less comorbidities than the included patients: $6.3 \%$ of these patients were known with COPD and $1.1 \%$ of these patients had a history of heart failure, the majority were women (72\%) and the mean age was 53 years. Following the YEARS algorithm, CTPA was 


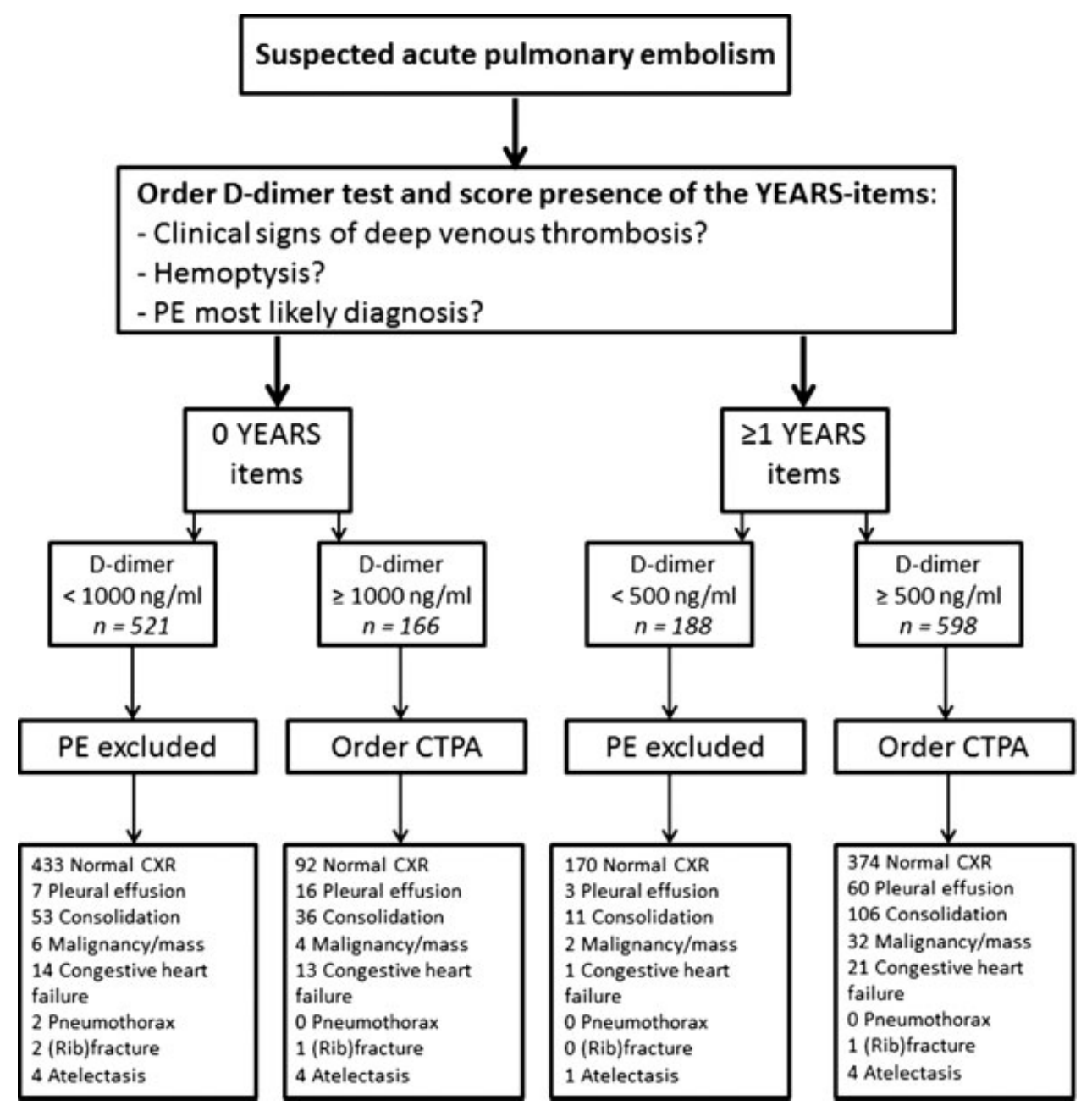

Fig. 1 The YEARS algorithm.

indicated to rule out or confirm the diagnosis of PE in 763 (52\%) of all patients. $P E$ was diagnosed in 214 patients at baseline for a prevalence of $15 \%$. Prevalence of PE among the 238 excluded patients without CXR was $17 \%$.

\section{CXR Results in All Patients}

The majority of patients had a normal CXR (73\%). Abnormal CXR was more frequent among patients with confirmed PE (36\%) than in patients without $\mathrm{PE}$ (26\%; - Table 2) for an OR of 1.60 (95\% CI: 1.18-2.18). Consolidation was the most frequent abnormality which was present in $23 \%$ of patients with PE versus $13 \%$ of patients without $\mathrm{PE}$ for an OR of 2.08 (95\% CI: 1.45-2.99). Other CXR abnormalities were quite similar between the group of patients with and without PE (-Table 2). The distribution of specific CXR results in different patient groups is illustrated in -Fig. $\mathbf{2}$.

\section{CXR Results in Patients with an Indication for CTPA}

From the 763 patients with an indication for CTPA according to the YEARS algorithm, the CXR was normal in 465 patients (61\%) compared with 604 of the 710 patients (85\%) in whom CTPA was not indicated for an OR of 3.65 (95\% CI: 2.84-4.70). Consolidation was the most frequent abnormality found on CXR in all four different YEARS groups (- Fig. 2).

\section{Pre- and Posttest Probability after CXR}

- Table 3 illustrates the different LRs with $95 \% \mathrm{CI}$ for all predefined CXR abnormalities. Pneumothorax and rib fracture were rare, with prevalence of only 0.1 and $0.3 \%$, respectively. For the overall population, only these two rare findings significantly lowered the posttest probability of PE with a positive LR of 0.00 ( - Table 3). Most of the other LRs were around 1.00, indicating that the result of the CXR did not change the posttest probability of a PE diagnosis. For patients with an indication for CTPA, only the CXR finding of a rib fracture, which was present in two patients, lowered the posttest probability to such an extent that CTPA could have been avoided ( - Table 3 ). Atelectasis on the CXR in patients with an indication for CTPA lowered the posttest probability on PE with a LR of 0.37 , although with a broad $95 \% \mathrm{CI}(0.05-3.0)$, consistent with an $8 \%(1 / 13)$ prevalence of PE in patients with atelectasis.

\section{Discussion}

In our cohort of patients with suspected PE, CXR was more frequently abnormal in patients who were diagnosed with PE than in those in whom PE was ruled out. The posttest probability of PE was only relevantly changed in patients with a (rib) fracture and/or a pneumothorax, which were 
Table 1 Baseline characteristics

\begin{tabular}{|l|l|}
\hline & $\begin{array}{l}\text { All patients } \\
(\boldsymbol{n}=1,473)\end{array}$ \\
\hline Mean age, y (SD) & $54.4(18.6)$ \\
\hline Female sex, $n(\%)$ & $922(62.6)$ \\
\hline Pulmonary embolism, $n(\%)$ & $214(14.5)$ \\
\hline CTPA indicated following YEARS, $n(\%)$ & $763(51.8)$ \\
\hline Prior VTE, $n(\%)$ & $146(9.9)$ \\
\hline COPD, $n(\%)$ & $208(14.1)$ \\
\hline Heart failure, $n(\%)$ & $30(2.0)$ \\
\hline Malignancy, $n(\%)$ & $133(9.0)$ \\
\hline Immobilization or recent surgery, $n(\%)$ & $159(10.8)$ \\
\hline Use of estrogen in women, $n(\%)$ & $131(14.2)$ \\
\hline Smoking, $n(\%)$ & $250(23.8)$ \\
\hline Symptoms, $n(\%)$ & \\
\hline Dyspnea & $1,045(70.9)$ \\
\hline Coughing & $579(39.3)$ \\
\hline Thoracic pain & $1,086(73.7)$ \\
\hline Palpitations & $115(7.8)$ \\
\hline Fever $\left(>38.5^{\circ} \mathrm{C}\right)$ & $47(3.2)$ \\
\hline
\end{tabular}

Abbreviations: COPD, chronic obstructive pulmonary disease; CTPA, computed tomography pulmonary angiography; VTE, venous thromboembolism.

rare findings. The incremental diagnostic value of CXR to the YEARS algorithm to rule out PE was therefore limited.

Previous studies have shown conflicting results for abnormalities on CXR observed in patients with PE. Two studies reported cardiomegaly as most common abnormality with a prevalence of 38\% (19/50 patients) and 27\% (622/2,315 patients), respectively. ${ }^{6,10}$ Robin et al found interstitial lung disease or consolidation as most prevalent abnormality (28\%) 11 and two other retrospective studies, of which one was the PIOPED study, reported atelectasis/parenchymal areas with increased opacity as most common abnormality with a prevalence of $68 \%{ }^{7,12}$ This heterogeneity in CXR findings demonstrates that a suspicion of acute PE may cause different nonspecific abnormalities on CXR. Considering this, the diagnostic value of CXR for the diagnosis of PE is therefore poor.

In the past, CXR was used as standard imaging test in the approach of patients with suspected PE to find alternative diagnosis and as a useful tool for the interpretation of the ventilation/perfusion scan (V/Q scan). ${ }^{7,11,12}$ Nowadays, CTPA is the first-choice imaging test in the diagnostic workup for patients with suspected PE due to the ability to directly visualize emboli, as well as alternative diagnosis. CXR is not needed for interpretation of the CTPA. Reasons why CXR is often used in clinical practice are its wide availability, the fast execution, the low radiation exposure compared with CTPA or VQ scan, and the low costs. ${ }^{14}$ Patients without an indication for CTPA, and thus a lower probability on PE, had more often a normal CXR in our cohort than patients referred for CTPA. However, normal CXR as well as abnormal CXR could not distinguish patients with from those without CTPA indication, nor could CXR distinguish between patients with or without PE.

CXR may have two different roles in the diagnostic workup of patients with suspected PE. First of all, CXR is an important diagnostic modality at the emergency department for the initial assessment of patients with respiratory and/or chest symptoms. The result of the CXR could lead to change suspected PE to another diagnosis or to moving PE higher up in the differential diagnosis. Moreover, the results of CXR, which were likely available for some of the patients when the physician completed the YEARS algorithm, could have led to assigning less or more YEARS items to the patient. This may have influenced the final YEARS classification and associated D-dimer threshold. For those reasons, CXR could therefore have contributed to the efficiency of the YEARS algorithm. ${ }^{5}$ Second, the CXR may be used as a routine test to exclude alternative diagnosis before performing a CTPA.

A limitation of this study is that because of the retrospective design and the lack of detailed information on the differential diagnosis of each individual patient, we were not able to explore the reason why the CXR was ordered, especially because CXR is no longer recommended nor considered among the useful tools for this specific clinical setting in recent guidelines. Also, for unknown reasons, not all patients in our cohort were referred for CXR which may have caused selection bias. Even so, CXR results hardly influenced the posttest probability of PE in any of the YEARS

Table 2 Overview of CXR findings in different patient groups

\begin{tabular}{|l|l|l|l|l|}
\hline Result of CXR & All patients $(\boldsymbol{n}=\mathbf{1 , 4 7 3})$ & PE $(\boldsymbol{n}=\mathbf{2 1 4})$ & No PE $(\boldsymbol{n}=\mathbf{1 , 2 5 9})$ & CTPA indicated $(\boldsymbol{n}=\mathbf{7 6 3})$ \\
\hline Normal CXR, $n(\%)$ & $1,069(72.6)$ & $137(64.0)$ & $932(74.0)$ & $465(60.9)$ \\
\hline Pleural effusion, $n(\%)$ & $86(5.8)$ & $14(6.5)$ & $72(5.7)$ & $76(10.0)$ \\
\hline Consolidation, $n(\%)$ & $206(14.0)$ & $49(22.9)$ & $157(12.5)$ & $142(18.6)$ \\
\hline Malignancy/mass, $n(\%)$ & $44(3.0)$ & $6(2.8)$ & $38(3.0)$ & $36(4.7)$ \\
\hline Congestive heart failure, $n(\%)$ & $49(3.3)$ & $7(3.3)$ & $42(3.3)$ & $34(4.5)$ \\
\hline Pneumothorax, $n(\%)$ & $2(0.1)$ & $0(0.0)$ & $2(0.2)$ & $0(0.0)$ \\
\hline (Rib) fracture, $n(\%)$ & $4(0.3)$ & $0(0.0)$ & $4(0.3)$ & $2(0.3)$ \\
\hline Atelectasis, $n(\%)$ & $13(0.9)$ & $1(0.5)$ & $12(1.0)$ & $8(1.0)$ \\
\hline
\end{tabular}

Abbreviations: CTPA, computed tomography pulmonary angiography; CXR, chest X-ray; PE, pulmonary embolism. 
No CTPA indication
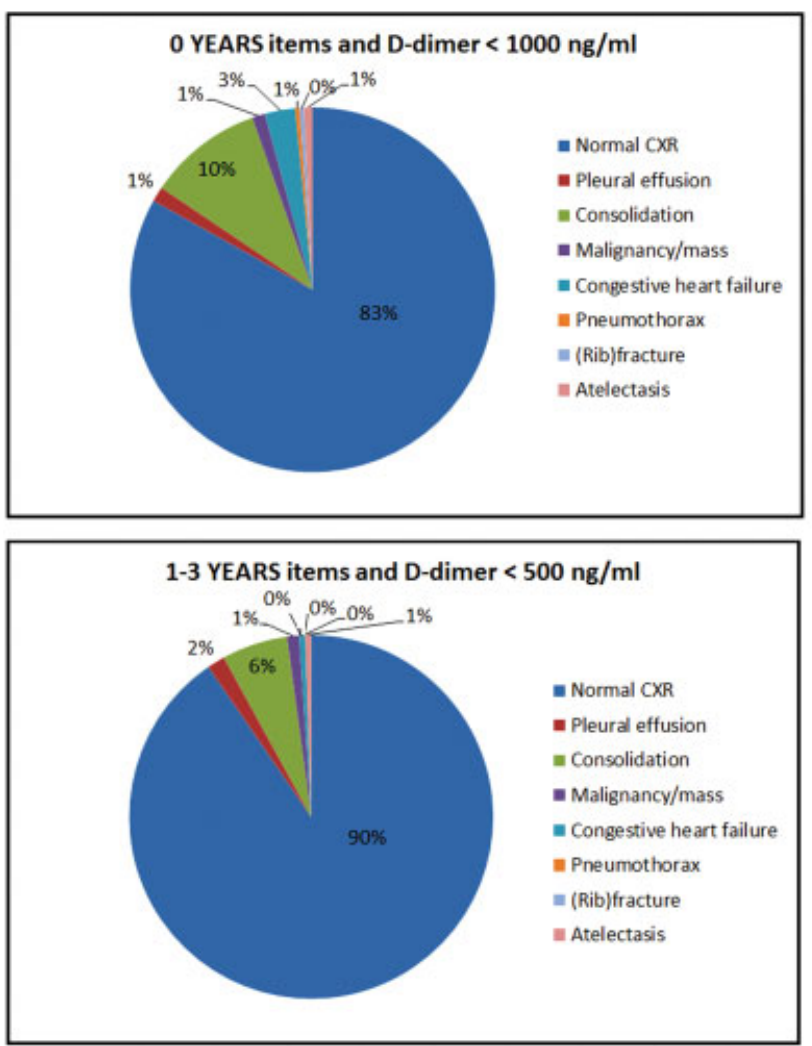

CTPA indication
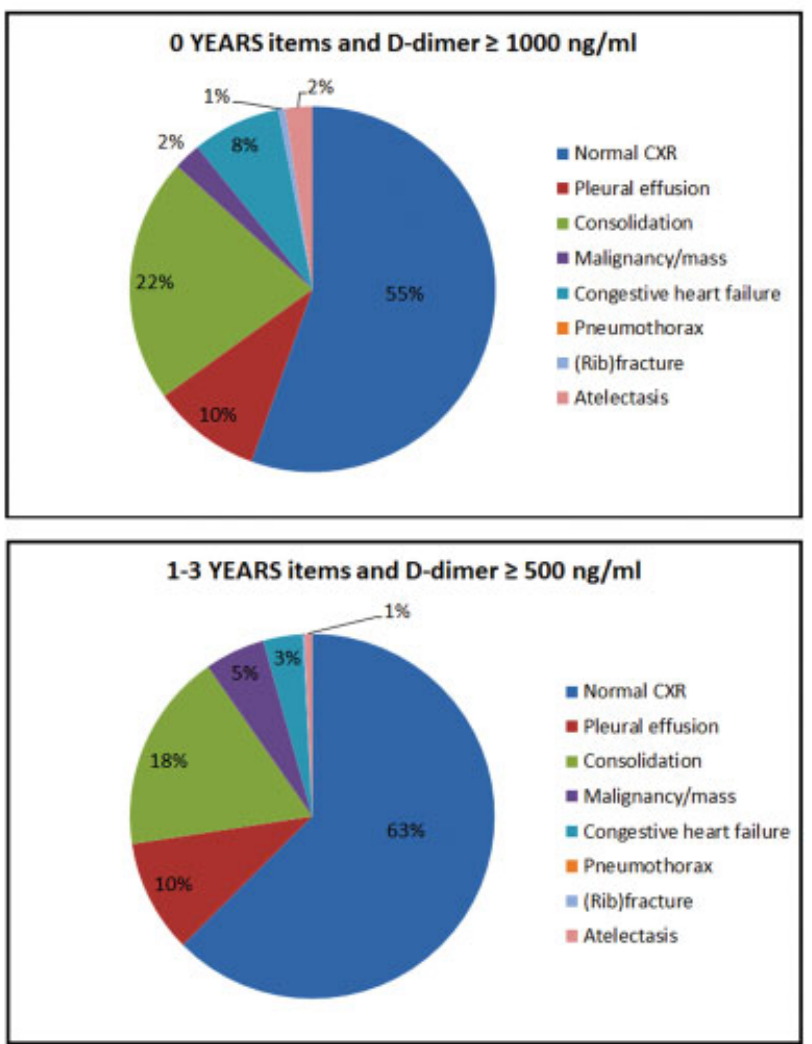

Fig. 2 CXR findings per YEARS group.

Table 3 Overview of LRs and CXR results in two groups; all patients and patients in whom CTPA was indicated according to the YEARS algorithm

\begin{tabular}{|l|l|l|l|l|}
\hline & \multicolumn{2}{|l|}{ All patients $(n=1,473)$} & \multicolumn{2}{l|}{$\begin{array}{l}\text { Patients in whom CTPA was indicated } \\
\text { according to the YEARS algorithm } \\
(\boldsymbol{n}=763)\end{array}$} \\
\hline Results CXR & Positive LR $(95 \% \mathrm{Cl})$ & Negative LR $(95 \% \mathrm{Cl})$ & Positive LR $(95 \% \mathrm{CI})$ & $\mathrm{Negative} \mathrm{LR} \mathrm{(95 \%} \mathrm{CI)}$ \\
\hline Normal CXR & $0.86(0.78-0.96)$ & $1.4(1.1-1.7)$ & $1.1(0.95-1.2)$ & $0.89(0.73-1.1)$ \\
\hline Pleural effusion & $1.1(0.66-2.0)$ & $0.99(0.95-1.0)$ & $0.58(0.33-1.0)$ & $1.1(1.0-1.1)$ \\
\hline Consolidation & $1.8(1.4-2.4)$ & $0.88(0.82-0.95)$ & $1.4(0.99-1.8)$ & $0.93(0.86-1.0)$ \\
\hline Malignancy/mass & $0.93(0.40-2.2)$ & $1.0(0.98-1.0)$ & $0.51(0.22-1.2)$ & $1.0(1.0-1.1)$ \\
\hline Congestive heart failure & $0.98(0.45-2.2)$ & $1.0(0.97-1.0)$ & $0.67(0.29-1.5)$ & $1.0(0.99-1.1)$ \\
\hline Pneumothorax & 0.00 & $1.0(0.99-1.0)$ & n.a. & n.a. \\
\hline (Rib) fracture & 0.00 & $1.0(0.99-1.0)$ & 0.00 & $1.0(0.99-1.0)$ \\
\hline Atelectasis & $0.49(0.06-3.8)$ & $1.0(0.99-1.0)$ & $0.37(0.05-3.0)$ & $1.0(0.99-1.0)$ \\
\hline
\end{tabular}

Abbreviations: $\mathrm{Cl}$, confidence interval; CTPA, computed tomography pulmonary angiography; CXR, chest X-ray; LR, likelihood ratio; n.a., not applicable; PE, pulmonary embolism.

Example: Assuming that the pretest probability of PE is $28 \%$ in a certain patient with suspected PE and an indication for CTPA according to YEARS, the posttest probability of PE in case of a normal CXR result would be $28 \% \times 1.1=31 \%$. The posttest probability of PE in this patient with any abnormality on CXR would be $28 \% \times 0.89=25 \%$.

categories. Therefore, our data do not support routine use of CXR in all patients before CTPA. Also, for unknown reasons, not all patients in our cohort were referred for CXR which may have caused selection bias.

In conclusion, CXR shows nonspecific abnormalities in patients with confirmed PE more frequently than in patents with PE ruled out. Only the rare findings of a (rib) fracture or pneumothorax significantly lowered the posttest probability to such an extent that CTPA could have been avoided according to the YEARS algorithm. Although CXR may play an important role in the initial diagnostic management in patients with suspected PE, our data do not support routine 
CXR in all patients with suspected PE, especially not in patients with an established indication for CTPA by YEARS.

\section{Authors' Contributions}

L.M.v.d.P. and F.A.K. had full access to all data in the study and take the responsibility for the integrity of the data and the accuracy of the data analysis.

Acquisition of the data: L.M.v.d.P., L.M.F., T.v.d.H., A.T.A.M., M.V.H., F.A.K.

Analysis and interpretation of the data: L.M.v.d.P., C.T., F.A.K. Image acquisition: L.J.M.K., A.d.R.

Drafting of the manuscript: L.M.v.d.P., C.T., F.A.K.

Critical revision of the manuscript: L.M.v.d.P., C.T., L.M.F., T.v.d.H., L.J.M.K., A.T.A.M., A.d.R., M.V.H., F.A.K.

Final approval of the manuscript: L.M.v.d.P., C.T., L.M.F., T. v.d.H., L.J.M.K., A.T.A.M., A.d.R., M.V.H., F.A.K.

\section{Conflict of Interest}

None declared.

\section{Acknowledgments}

Cecile Tromeur is supported by "Fonds de Dotation" "Recherche en Santé Respiratoire" et "Fondation du Souffle" and by "Institut Archipel” Brest, France.

\section{References}

1 Konstantinides SV. 2014 ESC Guidelines on the diagnosis and management of acute pulmonary embolism. Eur Heart J 2014;35 (45):3145-3146

2 Raja AS, Greenberg JO, Qaseem A, Denberg TD, Fitterman N, Schuur JD; Clinical Guidelines Committee of the American College of Physicians. Evaluation of patients with suspected acute pulmonary embolism: Best Practice Advice from the Clinical Guidelines Committee of the American College of Physicians. Ann Intern Med 2015;163(09):701-711
3 Huisman MV, Klok FA. How I diagnose acute pulmonary embolism. Blood 2013;121(22):4443-4448

4 Righini M, Van Es J, Den Exter PL, et al. Age-adjusted D-dimer cutoff levels to rule out pulmonary embolism: the ADJUST-PE study. JAMA 2014;311(11):1117-1124

5 van der Hulle T, Cheung WY, Kooij S, et al; YEARS Study Group. Simplified diagnostic management of suspected pulmonary embolism (the YEARS study): a prospective, multicentre, cohort study. Lancet 2017;390(10091):289-297

6 Zubairi AB, Husain SJ, Irfan M, Fatima K, Zubairi MA, Islam M. Chest radiographs in acute pulmonary embolism. J Ayub Med Coll Abbottabad 2007;19(01):29-31

7 Worsley DF, Alavi A, Aronchick JM, Chen JT, Greenspan RH, Ravin CE. Chest radiographic findings in patients with acute pulmonary embolism: observations from the PIOPED Study. Radiology 1993; 189(01):133-136

8 Bergus GR, Barloon TS, Kahn D. An approach to diagnostic imaging of suspected pulmonary embolism. Am Fam Physician 1996;53 (04):1259-1266

9 Coche E, Verschuren F, Hainaut P, Goncette L. Pulmonary embolism findings on chest radiographs and multislice spiral CT. Eur Radiol 2004;14(07):1241-1248

10 Elliott CG, Goldhaber SZ, Visani L, DeRosa M. Chest radiographs in acute pulmonary embolism. Results from the International Cooperative Pulmonary Embolism Registry. Chest 2000;118(01): 33-38

11 Robin P, Le Roux PY, Tissot V, et al. Interest of chest X-ray in tailoring the diagnostic strategy in patients with suspected pulmonary embolism. Blood Coagul Fibrinolysis 2015;26(06):643-648

12 Srivastava SD, Eagleton MJ, Greenfield LJ. Diagnosis of pulmonary embolism with various imaging modalities. Semin Vasc Surg 2004;17(02):173-180

13 National Clinical Guideline Centre (UK). Venous thromboembolic diseases: the management of venous thromboembolic diseases and the role of thrombophilia testing. London: Royal College of Physicians (UK); June 2012

14 Cardinale L, Volpicelli G, Lamorte A, Martino J, Veltri A. Revisiting signs, strengths and weaknesses of standard chest radiography in patients of acute dyspnea in the emergency department. J Thorac Dis 2012;4(04):398-407 